\title{
Trial Statistician
}

National Cancer Institute

\section{Source}

National Cancer Institute. Trial Statistician. NCI Thesaurus. Code C142737.

A statistician who has a combination of education, training and experience to be responsible for the statistical aspects of the trial. (ICH) 